J. K. Verma

Nagoya Math. J.

Vol. 118 (1990), 155-163

\title{
JOINT REDUCTIONS OF COMPLETE IDEALS
}

\author{
J. K. VERMA
}

\section{§1. Introduction}

The aim of this paper is to extend and unify several results concerning complete ideals in 2-dimensional regular local rings by using the theory of joint reductions and mixed multiplicities. The theory of complete ideals in a 2-dimensional regular local ring was developed by Zariski in his 1938 paper [Z]. This theory is presented in a simpler and general form in [ZS, Appendix 5] and [H2].

The Zariski's product theorem asserts that the product of complete ideals in a 2-dimensional regular local ring is again complete. Counterexamples to such a statement in 3-dimensional regular local rings have been given by Huneke in $[\mathrm{H} 1, \S 3]$. A surprising generalization of Zariski's product theorem was obtained by Huneke and Sally in [H-S, Theorem 4.1]; Let $(R, m)$ be a $d$-dimensional Cohen-Macaulay local ring satisfying $\left(R_{2}\right)$. Let $I$ be a height two complete ideals of analytic spread two. Then $I^{k}$ is complete for all positive integers $k$. Inspired by this theorem, Huneke asked the following

Question. Let $I$ and $J$ be height two complete ideals of analytic spread two in a $d$-dimensional Cohen-Macaulay local ring satisfying $\left(R_{2}\right)$. Suppose that $I J$ has analytic spread two. Is it true that $I J$ is complete?

We shall use joint reductions to prove that Huneke's question has an affirmative answer. For this purpose we shall prove our main theorem 2.1 in $\S 2$ that if $I$ and $J$ are $m$-primary complete ideals in a two dimensional regular local ring $(R, m)$ with infinite residue field then there exists elements $a \in I$ and $b \in J$ such that $a J+b I=I J$. By taking $I=J$ we obtain the Lipman-Teisier theorem: $I^{2}=(a, b) I$, which they proved in [L-T, Corollary 5.4].

Inspired by the equation $a J+b I=I J$ in $\S 3$, we study the Bhattacharya function $B(r, s)=$ length $\left(R / I^{r} J^{s}\right)$ for $m$-primary ideals

Received February 21, 1989. 
$I$ and $J$ in a two dimensional Cohen-Macaulay local ring $R$. Bhattacharya showed in [B] that there is a polynomial $P(r, s)$ of total degree 2 in $r$ and $s$ such that $B(r, s)=P(r, s)$ for all large values of $r$ and $s$. Let $e_{1}(I \mid J)$ denote the coefficient of $r s$ in $P(r, s)$. If $I$ and $J$ are complete $m$-primary ideals in a two dimensional regular local ring $R$ then it follows by a result of Lipman [L, Corollary 3.7] that

$$
e_{1}(I \mid J)=l(R / I J)-l(R / I)-l(R / J),
$$

where $l$ denotes length. As a consequence of our main theorem 2.1 we deduce this formula. We note that our treatment of $\left(^{*}\right)$ is much simpler since Lipman's proof makes use of the theory of point bases of ideals and a formula of Hoskin and Deligne which in turn is proved in [L] by using sheaf cohomology.

For techniques and methods of proof we often use Huneke's lucid exposition of theory of complete ideals in a two dimensional regular local ring [H2] and Huneke and Sally's paper [H-S]. Our sole contribution is a careful blend of joint reductions and mixed multiplicities in their proofs thereby achieving a unification of many beautiful theorems about complete ideals.

\section{$\S 2$. The main theorem}

In this section we prove our main theorem concerning joint reductions of complete ideals in a two dimensional regular local ring. We recall the concept of joint reductions introduced by Rees in [R1]. Let $I_{1}, \cdots, I_{r}$ be ideals of a ring $R$. A set of elements $\left(x_{1}, \cdots, x_{r}\right)$ with $x_{1} \in$ $I_{1}, \cdots, x_{r} \in I_{r}$ is called a joint reduction of the set of ideals $\left(I_{1}, \cdots, I_{r}\right)$ if there exists positive integers $a_{1}, \cdots, a_{r}$ so that

$$
I_{1}^{a_{1}} I_{2}^{a_{2}} \cdots I_{r}^{a_{r}}=\sum_{i=1}^{r} x_{i} I_{1}^{a_{1}} \cdots I_{i}^{a_{i}-1} \cdots I_{r}^{a_{r}}
$$

In our main theorem 2.1 we show that if $I$ and $J$ and complete $m$ primary ideals of a two dimensional regular local ring and $(a, b)$ is a joint reduction of the set $(I, J)$ then $I J=a J+b I$. For this purpose we need to recall several facts from Zariski's theory of complete ideals. Let $(R, m)$ be a two dimensional regular local ring. An ideal $I$ is called contracted if there exists $x \in m \backslash m^{2}$ such that $I=I R[m / x] \cap R$. We say that $I$ is contracted from $R[m / x]$. By Lemma 2 of [ZS, Appendix 5] $I$ is 
contracted from $R[m / x]$ if and only if $I: m=I: x$ and by Lemma 3 of [loc. cit.] if $I$ and $J$ are contracted from $R[m / x]$ then so is $I J$. Rees [R2] and Lipman [L, Corollary 3.2] showed that if an $m$-primary ideal $I$ is contracted then $\mu(I)=1+\operatorname{ord}(I)$ where $\mu(I)=l(I / m I)$ and $\operatorname{ord}(I)=$ $\max \left\{n \mid I \subset m^{n}\right\}$. Huneke and Sally proved [H-S, Theorem 2.1] that if $\mu(I)=1+\operatorname{ord}(I)$ then $I$ is contracted provided $R / m$ is infinite.

Let $I$ be an $m$-primary ideal contracted from $S=R[m / x]$. If $N$ is any maximal ideal of $S$ containing $m S$ then $S_{N}$ is a 2-dimensional regular local ring. If $\operatorname{ord}(I)=r$ then $I S=x^{r} I^{\prime} S$ for an ideal $I^{\prime}$ in $S . I^{\prime}$ is called transform of $I$ in $S$ and $I^{\sim}=I_{N}^{\prime}$ is called tranform of $I$ in $S_{N}$. By Proposition 5 of [ZS, Appendix 5], if $I$ is complete then $I^{\prime}$ and hence $I^{\sim}$ are complete. A complete ideal is contracted [H2, Proposition 3.1]. Finally, by Proposition 3.6 of [H2], $e(I)>e\left(I^{\sim}\right)$ where $e$ denotes multiplicity.

THEOREM 2.1. Let $(R, m)$ be a 2-dimensional regular local ring. Let I and $J$ be complete m-primary ideals. Then for any joint reduction $(a, b)$ of $(I, J) ; a J+b I=I J$.

Proof. First we remark that if $R / m$ is infinite then joint reductions do exist by [R1]. We prove this theorem by induction on $t=\max (e(I)$, $e(J))$. If $t=1$ then $e(I)=e(J)=1$. We may assume without loss of generality that $R / m$ is infinite. Then there exist elements $c, d$ in $I$ such that $(c, d)$ is a reduction of $I$, i.e., $(c, d) I^{n}=I^{n+1}$ for some $n$ by [NR]. It follows that $e((c, d))=e(I)$. Since $R$ is regular, it is Cohen-Macaulay. Hence $e(I)=e((c, d))=l(R /(c, d))=1=l(R / m)$. Thus $m=(c, d)=I$. Similarly $J=m$. In this case $m^{2}=c m+d m$. Suppose that the theorem has been proved for all positive integers less than $t$. Let $(a, b)$ be a joint reduction of $(I, J)$. We show that the ideal $K=a J+b I$ is contracted.

There exists an $n$ so that $a I^{n-1} J^{n}+b I^{n} J^{n-1}=I^{n} J^{n}$. This implies that $a$ (resp. $b$ ) is part of a minimal basis of $I$ (resp. $J$ ). Indeed, if $a \in m I$ then $I^{n} J^{n}=a I^{n-1} J^{n}+b I^{n} J^{n-1} \subset m I^{n} J^{n}+b I^{n} J^{n-1} \subset I^{n} J^{n}$. Thus $I^{n} J^{n}=$ $m I^{n} J^{n}+b I^{n} J^{n-1}$. By Nakayama's lemma, $b I^{n} J^{n-1}=I^{n} J^{n}$. This implies that (b) is a reduction of $I$ which is a contradiction since $I$ is $m$-primary. Similarly $b$ is part of a minimal basis of $J$. Put ord $(I)=r$ and $\operatorname{ord}(J)=s$. Since $I$ and $J$ are complete, they are contracted. Hence $\mu(I)=r+1$ and $\mu(J)=s+1$. choose $a_{1}, a_{2}, \cdots, a_{r} \in I$ and $b_{1}, b_{2}, \cdots, b_{s} \in J$ such that $I=\left(a_{0}, a_{1}, \cdots, a_{r}\right)$ and $J=\left(b_{0}, b_{1}, \cdots, b_{s}\right)$ where $a=a_{0}$ and $b=b_{0}$. Then 


$$
K=\left(a_{0} b_{0}, a_{0} b_{1}, \cdots, a_{0} b_{s}, b_{0} a_{1}, b_{0} a_{2}, \cdots, b_{0} a_{r}\right) .
$$

We show that the generators of $K$ displayed in (*) form a minimal basis of $K$. Suppose that we have a relation

$$
\sum_{i=0}^{s}\left(a_{0} b_{i}\right) u_{i}+\sum_{j=1}^{r}\left(b_{0} a_{j}\right) v_{j}=0
$$

where $u_{0}, \cdots, u_{s}, v_{1}, v_{2}, \cdots, v_{r} \in R$. Since $(a, b)$ is a joint reduction of $(I, J),(a, b)$ is $m$-primary. Consequently $a$ and $b$ are coprime. Thus there exists an $f \in R$ such that

$$
a_{0} f=\sum_{j=1}^{r} a_{j} v_{j} \quad \text { and hence } \quad b_{0} f=\sum_{i=0}^{s} b_{i} u_{i} .
$$

This implies that $f, v_{1}, v_{2}, \cdots, v_{r} \in m$ and $u_{0}-f, u_{1}, \cdots, u_{s} \in m$. Consequently the generators displayed in (*) form a minimal basis of $K$. Since $(a, b)$ is a joint reduction of $(I, J)$, there exists $n$ such that $(a J+$ $b I)(I J)^{n}=(I J)^{n+1}$. Hence $\operatorname{ord}(a J+b I)=\operatorname{ord}(I J)=r+s$. Thus $\mu(a J+$ $b I)=1+r+s$ which implies that $a J+b I$ is contracted. We may assume that $K$ and $I J$ are contracted from $S=R[m / x]$. Write $a=a^{\prime} x^{r}$ and $b=$ $b^{\prime} x^{s}$ for some $a^{\prime}, b^{\prime} \in S$ and $I=x^{r} I^{\prime} S$ and $J=x^{s} J^{\prime} S$. Then

$$
K S=\left(a^{\prime} J^{\prime}+b^{\prime} I^{\prime}\right) x^{r+s} S \text { and } I J S=x^{r+s} I^{\prime} J^{\prime} S .
$$

Thus $\left(a^{\prime}, b^{\prime}\right)$ is a joint reduction of $\left(I^{\prime}, J^{\prime}\right)$. Hence it is enough to prove that $a^{\prime} J^{\prime}+b^{\prime} I^{\prime}=I^{\prime} J^{\prime}$ since $I J$ and $K$ are contracted. To prove that the last equation holds we may localize at any maximal ideal $N$ containing $a^{\prime} J^{\prime}+b^{\prime} I^{\prime}$. By Huneke's theorem $e\left(I^{\prime} S_{N}\right)<e(I)$ and $e\left(J^{\prime} S_{N}\right)<e(J)$. Thus by induction hypothesis $\left(a^{\prime} J^{\prime}+b^{\prime} I^{\prime}\right) S_{N}=I^{\prime} J^{\prime} S_{N}$ for all $N$ containing $a^{\prime} J^{\prime}+b^{\prime} I^{\prime}$. Hence $a^{\prime} J^{\prime}+b^{\prime} I^{\prime}=I^{\prime} J^{\prime}$ and consequently $I J=a J+b I$.

CoRollary 2.2 (Lipman-Teissier). Let $(R, m)$ be a 2-dimensional regular local ring. Then for any reduction $(a, b)$ of an m-primary complete ideal $I,(a, b) I=I^{2}$.

Proof. If $(a, b)$ is a reduction of $I$ then there is an $n$ such that $(a, b) I^{n}=I^{n+1}$. Hence $(a, b) I^{2 n+1}=I^{2 n+2}$ which can be rewritten as $a I^{n} I^{n+1}$ $+b I^{n} I^{n+1}=I^{n+1} I^{n+1}$. Thus $(a, b)$ is a joint reduction of the set $(I, I)$. Hence $a I+b I=I^{2}$ which completes the proof. 


\section{§3. Applications of the main theorem}

In this section we study the Bhattacharya polynomial of two $m$ primary ideals in a 2-dimensional Cohen-Macaulay local ring. By combining this with our main theorem we give simpler proofs of several results about complete ideals in a 2-dimensional regular local ring.

Let $(R, m)$ be a two dimensional local ring. For $m$-primary ideals $I$ and $J$ consider the function $B(r, s)=l\left(R / I^{r} J^{s}\right)$. By [B], there exists a polynomial $P(r, s)$ with rational coefficients such that for large $r$ and $s$, $P(r, s)=B(r, s)$. The polynomial $P(r, s)$ is called the Bhattacharya polynomial of $I$ and $J$. The total degree of $P(r, s)$ is 2 and it can be written in the form

$$
P(r, s)=e(I)\left(\begin{array}{l}
r \\
s
\end{array}\right)+e_{1}(I \mid J) r s+e(J)\left(\begin{array}{l}
s \\
2
\end{array}\right)+f r+g s+h,
$$

where $e_{1}(I \mid J)$ is a positive integer called the mixed multiplicity of $I$ and $J$ and $f, g, h$ are integers. By a theorem of Rees in [R1], for any joint reduction $(a, b)$ of $(I, J), e((a, b))=e_{1}(I \mid J)$. We begin with a lemma.

Lemma 3.1. Let $(R, m)$ be a local ring of dimension $\geq 2$. Let $I$ and $J$ be ideals of $R$. If $a \in I$ and $b \in J$ are such that $(a, b)$ is an $R$-sequence, then the R-module homomorphism

$$
f: R / I \oplus R / J \longrightarrow(a, b) /(a J+b I)
$$

defined as $f(\bar{x}, \bar{y})=(x b+y a)+(a J+b I)$ is an isomorphism.

Proof. It is clear that $f$ is a surjective $R$-module homomorphism. Suppose that $x b+y a \in a J+b I$. Then $x b \in(a, b I)$. Choose $c \in R$ and $d \in I$ so that $x b=c a+b d$. Hence $b(x-d)=c a$ which gives $x-d \epsilon$ $(a: b)=(a)$. Thus $x \in I$. Similarly $y \in J$. Hence $f$ is injective.

Let $I$ and $J$ be complete $m$-primary ideals in a two dimensional regular local ring $R$. By a result of Lipman [L. Corollary 3.7]

$$
e_{1}(I \mid J)=l(R / I J)-l(R / I)-l(R / J) .
$$

In our next result we generalize that above "mixed multiplicity formula of Lipman" to 2-dimensional Cohen-Macaulay local rings.

Theorem 3.2. Let $(R, m)$ be a two dimensional Cohen-Macaulay local ring with $R / m$ infinite. Let $I$ and $J$ be m-primary ideals. Then the following are equivalent: 
(a) There exist $a \in I$ and $b \in J$ with $a J+b I=I J$.

(b) For all integers $r, s \geq 1 ; a^{r} J^{s}+b^{s} I^{r}=I^{r} J^{s}$.

(c) $l\left(R / I^{r} J^{r}\right)=l\left(R / I^{r}\right)+r s e_{1}(I \mid J)+l\left(R / J^{s}\right)$ for all $r, s$.

(d) $e_{1}(I \mid J)=l(R / I J)-l(R / I)-l(R / J)$.

Proof. (a) $\Rightarrow$ (b). By symmetry, it is enough to show that $a^{r} J+b I^{r}$ $=I^{r} J$ for all $r$. Use induction on $r$. Assume that this equation holds for $r$. Then

$$
\begin{aligned}
I^{r+1} J & =I\left(I^{r} J\right) \\
& =I\left(a^{r} J+b I^{r}\right) \\
& =a^{r} I J+b I^{r+1} \\
& =a^{r}(a J+b I)+b I^{r+1} \\
& =a^{r+1} J+b I^{r+1} .
\end{aligned}
$$

(b) $\Rightarrow$ (c). By the lemma 3.1 we get

$$
R / I^{r} \oplus R / J^{s} \simeq\left(a^{r}, b^{s}\right) / a^{r} J^{s}+b^{s} I^{r}
$$

since the equation $a^{r} J^{s}+b^{s} I^{r}=I^{r} J^{s}$ forces $\left(a^{r}, b^{s}\right)$ to be an $R$-sequence in view of the Cohen-Macaulayness of $R$. Hence for all $r, s \geq 1$

$$
l\left(R / I^{r}\right)+l\left(R / J^{s}\right)=l\left(R / I^{r} J^{s}\right)-l\left(R /\left(a^{r}, b^{s}\right)\right) .
$$

Since $R$ is Cohen-Macaulay, $l\left(R /\left(a^{r}, b^{s}\right)\right)=\operatorname{rsl}(R /(a, b))=\operatorname{rse}((a, b))=$ $r e_{1}(I \mid J)$ by [R1, Theorem 2.4]. Thus (c) follows.

(c) $\Rightarrow$ (d). Clear.

(d) $\Rightarrow$ (a). Since $R / m$ is infinite there exists a joint reduction $(a, b)$ of $(I, J)$ by [R1, Corollary (i)]. By Rees' theorem 2.4 in [R1], $e_{1}(I \mid J)=$ $e((a, b))=l(R /(a, b))$. By Lemma 3.1

$$
l(R / I)+l(R / J)=l(R / a J+b I)-l(R /(a, b)) .
$$

Hence $e_{1}(I \mid J)=l(R / a J+b I)-l(R / I)-l(R / J)$. Comparing this expression with the formula for $e_{1}(I \mid J)$ in (d) we conclude that $a J+b I=I J$.

Corollary 3.3 (Lipman). Let $(R, m)$ be a 2-dimensional regular local ring. Then for any m-primary complete ideals $I$ and $J$

$$
e_{1}(I \mid J)=l(R / I J)-l(R / I)-l(R / J) .
$$

Proof. By passing to $R[x]_{m R[x]}$ we may assume that $R / m$ is infinite. Now use Theorem 3.2 and Theorem 3.1. 
Corollary 3.4 (Lipman). Let $I$ be a complete m-primary ideal of a 2-dimensional regular local ring. Then for all $n$

$$
l\left(R / I^{n}\right)=e(I)\left(\begin{array}{c}
n+1 \\
2
\end{array}\right)-[e(I)-l(R / I)] n .
$$

Proof. By Corollary 3.3 and Theorem 3.2 we have

$$
l\left(R / I^{n}\right)=l\left(R / I^{n-1} I\right)=l\left(R / I^{n-1}\right)+(n-1) e_{1}(I \mid I)+l(R / I) .
$$

The assertion of the corollary is clearly true for $n=1$. Suppose it is true for $n-1$. Then by using $e_{1}(I \mid I)=e(I)$,

$$
\begin{aligned}
l\left(R / I^{n}\right) & =e(I)\left(\begin{array}{l}
n \\
2
\end{array}\right)-[(e(I)-l(R / I)](n-1)+(n-1) e(I)+l(R / I) \\
& =e(I)\left(\begin{array}{l}
n \\
2
\end{array}\right)+n e(I)-[e(I)-l(R / I)] n \\
& =e(I)\left(\begin{array}{c}
n+1 \\
2
\end{array}\right)-[e(I)-l(R / I)] n .
\end{aligned}
$$

Corollary 3.5. Let $(R, m)$ be a 2-dimensional regular local ring. Let $I$ and $J$ be m-primary complete ideals. Then for all $r, s, \geq 1$,

$$
l\left(R / I^{r} J^{s}\right)=e(I)\left(\begin{array}{l}
r \\
2
\end{array}\right)+r s e_{1}(I \mid J)+e(J)\left(\begin{array}{l}
s \\
2
\end{array}\right)+r l(R / I)+s l(R / J),
$$

where

$$
e_{1}(I \mid J)=l(R / I J)-l(R / I)-l(R / J) .
$$

Proof. By Theorem 3.2, Theorem 2.1 and Corollary 3.4 we get

$$
\begin{aligned}
l\left(R / I^{r} J^{s}\right)= & l\left(R / I^{r}\right)+r s e_{1}(I \mid J)+l\left(R / J^{s}\right) \quad \text { for all } r, s \\
= & e(I)\left(\begin{array}{c}
r+1 \\
2
\end{array}\right)-[e(I)-l(R / I)] r+r s e_{1}(I \mid J) \\
& +e(J)\left(\begin{array}{c}
s+1 \\
2
\end{array}\right)-[e(J)-l(R / J)] s \\
= & e(I)\left(\begin{array}{l}
r \\
2
\end{array}\right)+r s e_{1}(I \mid J)+e(J)\left(\begin{array}{l}
s \\
2
\end{array}\right)+r l(R / I)+s l(R / J) .
\end{aligned}
$$

\section{§4. On a question of Huneke}

In this section we answer a question of Huneke mentioned in the introduction. Recall that the analytic spread of an ideal $I$ in a local ring $(R, m)$ is, by definition, the dimension of the graded ring $R / m \oplus I / \operatorname{Im} \oplus$ $I^{2} / I^{2} m \oplus \cdots$. Analytic spread of $I$ is denoted by $a(I)$. It is well known 
that $h t I \leq a(I) \leq \mu(I)$. If $R / m$ is infinite then there is an ideal $J$ contained in $I$ so that $\mu(J)=a(I)$ and $J I^{n}=I^{n+1}$ for some $n$. We refer the reader to $[\mathrm{NR}]$ for details. An ideal is called equimultiple if $h t I=a(I)$. Note that all $m$-primary ideals are equimultiple. We remark that the proof of the next theorem differs from that of Theorem 4.1 of [H-S] only in the use of joint reductions instead of reductions.

Theorem 4.1. Let $(R, m)$ be a d-dimensional Cohen-Macaulay local ring satisfying $\left(R_{2}\right)$. Let $I$ and $J$ be height two equimultiple complete ideal so that $I J$ is also equimultiple. Then for all $r, s \geq 1, I^{r} J^{s}$ is a complete equimultiple ideal.

Proof. We may assume without loss of generality that $R / m$ is infinite. By [O] there exist $a \in I$ and $b \in J$ so that $a J+b I$ is a reduction of $I J$. It follows that $(a, b)$ is an $R$-sequence and hence by Lemma 3.1 we get

$$
\operatorname{Ass}(a, b) / a J+b I=\operatorname{Ass} R / I \cup \operatorname{Ass} R / J .
$$

By considering the exact sequence

$$
0 \longrightarrow(a, b) / a J+b I \longrightarrow R / a J+b I \longrightarrow R /(a, b) \longrightarrow 0
$$

we get $\operatorname{Ass}(R / a J+b I) \subset \operatorname{Ass} R /(a, b) \cup \operatorname{Ass} R / I \cup \operatorname{Ass} R / J$. By Proposition 4.1 of [Mc], $I$ and $J$ are unmixed ideals. Hence $a J+b I$ is an unmixed ideal. Suppose that $a J+b I<I J$. Then there exists a height 2 prime $P \in \operatorname{Ass}(R / a J+b I)$ so that $(a J+b I) R_{P}<I J R_{P}$. It follows that $P \subset I+J$. Since $R_{P}$ is regular, by Theorem $2.1, I J R_{P}=(a J+b I) R_{P}$. Hence $a J+b I$ $=I J$ and consequently $I J$ is unmixed. Thus $I J=\cap(I J) R_{P}$ where the intersection ranges over all associated primes of $I J$. By Zariski's product theorem $I J R_{P}$ is complete. Therefore $I J$ is complete. That $I^{r} J^{s}$ is equimultiple follows from Theorem 1 of [O] and Corollary (ii) of [R1]. The completeness of $I^{r} J^{s}$ follows by double induction on $r$ and $s$.

Acknowledgements. I would like to thank Professors W. Heinzer and C. Huneke from whom I learned this subject. Thanks are also due to Professor D. Katz for valuable discussions.

\section{REFERENCES}

[B] P. B. Bhattacharya, The Hilbert function of two ideals, Proc. Cambridge Philos. Soc., 53 (1957), 568-575.

[H1] C. Huneke, The primary components of and integral closures of ideals in 3-dimensional regular local rings, Math. Ann., 275 (1986), 617-635. 
[H2] C. Huneke, Complete ideals in two-dimensional regular local rings, proceedings of "Microprogram on Commutative Algebra" held at MSRI, Berkeley, CA (1987) Springer-Verlag, New York (1989).

[H-S] C. Huneke and J. D. Sally, Birational extensions in dimension two and integrally closed ideals, J. Algebra, 115 (1988), 481-500.

[L] J. Lipman, On complete ideals in regular local rings, Commutative Algebra and Algebraic Geometry: in honor of M. Nagata.

[L-T] J. Lipman and B. Teissier, Pseudo-rational local rings and a theorem of BriançonSkoda about integral closures of ideals, Michigan Math. J., 28 (1981), 97-116.

[Mc] S. McAdam, "Asymptotic prime divisors," Lecture notes in Mathematics, Vol. 1023, Springer-Verlag, Berlin, 1983.

[NR] D. G. Northcott and D. Rees, Reductions of ideals in local rings, Proc. Cambridge Philos. Soc., 50 (1954), 145-158.

[0] L. O'Carroll, On two theorems concerning reductions in local rings, J. Math. Kyoto Univ., 27 (1987), 61-67.

[R1] D. Rees, Generalizations of reductions and mixed multiplicities, J. London Math. Soc., (2) 29 (1984), 397-414.

[R2] D. Rees, Hilbert functions and pseudo-rational local rings of dimension 2, J. London Math. Soc., (2) 24 (1981), 467-479.

[Z] O. Zariski, Polynomial ideals defined by infinitely near points, Amer. J. Math., 60 (1938), 151-204.

[ZS] O. Zariski and P. Samuel, "Commutative Algebra," Vol. 2, van Nostrand, Princeton, 1960 .

Department of Mathematics

Indian Institute of Technology

Powai, Bombay-400076

India 